SELECTED PAPER AT NCSP'15

\title{
Validation of the Minimum-Error Method for Estimating Model Parameters from Neural Spike Train Data
}

\author{
Huu Hoang and Isao T. Tokuda \\ Graduate School of Science and Engineering, Ritsumeikan University \\ 1-1-1 Noji-higashi, Kusatsu-shi, Shiga 525-8577, Japan \\ E-mail: gr0119he@ed.ritsumei.ac.jp, isao@fc.ritsumei.ac.jp
}

\begin{abstract}
A minimum-error method was developed in our previous study to estimate model parameters from spike train data experimentally recorded from inferior olive neurons. Our method characterized the neuronal firing dynamics using 67 spatiotemporal features. The closest match between experimental and simulated data in the feature space determined two parameters that control the neural network dynamics of the simulation model. This approach, however, has not been fully verified, because the true parameter values were unknown for the experimental data. In the present study, we attempt to validate the minimum-error method using simulated spike data, for which the true parameter values are known, as the test data. Our performance evaluation on the test data confirmed that the minimum-error method is effective for resolving the inverse problem even when the simulation model is an imperfect representation of the experimental data.
\end{abstract}

\section{Introduction}

Advanced measurement technologies in neuroscience enable the collection of a massive amount of neuronal data. Nevertheless, exploring the underlying functional mechanisms is a very difficult task. For instance, given measured spike trains of a population of neurons, it is not trivial to understand how the neurons electrically interact with each other to induce synchronized or irregular firing patterns. Although mathematical modeling of the brain provides a plausible solution, it usually faces two challenges. First, the model should be constructed such that it is not only consistent with the anatomy of the neuronal network, but also capable of reproducing the quantitative dynamics of the brain. Second, an effective method for fitting the simulated spike data to the experimental data is strongly required $[1,2]$.

In the previous study [3], we targeted inferior olive (IO) neurons, which play a central role in cerebellar learning. The IO network model was composed of nine neurons receiving excitatory and inhibitory inputs. The nine neurons were connected to each other via gap junctions. In the forward mode, we characterized the IO firing dynamics by 67 features extracted from the spike data. We found that the simulated neurons did not perfectly reproduce the firing dynamics of the real neurons because of a huge mismatch in the system complexity between the model and the brain. We thus divided the experimental spike data into short time segments and estimated two model parameters for each time segment by minimizing the distance between the simulated and experimental spike data. Then, the distribution of the estimated parameter values from all segments was treated as a population of estimates. This simple algorithm worked fairly well, as indicated by the extremely small errors between the experimental and simulation spike data in the feature space. The estimates, however, have not yet been formally validated, because the true parameter values of the experimental data were not known. To enable its further application to other experimental data, validation of the previously developed method is strongly desired. Adopting the same task of estimating two conductance values in the IO network model, in this study, we utilize simulated spike data as the test data. Unlike the experimental data, the underlying conductance values are known for the simulation data, which are thus suitable for validation purpose. Here, three representative conductance values that display qualitatively different firing dynamics were selected to generate the test data. The results for the test data demonstrated that the minimum-error method is effective for resolving the inverse problem even in the presence of imperfectness of the model.

\section{Methods}

It has been shown that the gap-junctional $\left(g_{c}\right)$ and inhibitory $\left(g_{i}\right)$ conductance values are two major determinants of IO firing. The aim of our study is to estimate these two parameters from the spike data of the IO network model. As training data sets, the network model was simulated to generate spike trains for about 1000 combinations of $g_{i}$ and $g_{c}$. We also simulated spike data for three sets of distinctive model parameters as test data sets. The firing dynamics were characterized by applying principal component analysis (PCA) 
to the feature vectors of the spike data. The model parameters, the corresponding training data of which best fit the test data in the PCA space, were determined as the estimates. The absolute differences between the estimated and true parameter values were used to evaluate the performance of the minimum-error method.

\subsection{Network model simulation}

The simulation model was composed of $3 \times 3$ neurons, each of which consists of soma, dendrite, and spine compartments [3]. The neurons were connected to each other via gap junctions $\left(g_{c}\right)$. All compartments received excitatory $\left(g_{e}\right)$ and inhibitory $\left(g_{i}\right)$ inputs driven by Gaussian noise generators. Synaptic noise was added to produce the irregular spatiotemporal dynamics observed experimentally in IO spike trains. The two parameters, $g_{i}$ and $g_{c}$, were varied in the range of 0 to $1.5 \mathrm{mS} / \mathrm{cm}^{2}$ in increments of $0.05 \mathrm{mS} / \mathrm{cm}^{2}$.

\subsection{Test data sets}

For three representative model parameter sets of $\left(g_{i}, g_{c}\right)$ $=(0.4,1.4)(\sharp 1),(1.4,1.4)(\sharp 2)$, and $(1.4,0.4)(\sharp 3)$, we simulated the test spike data for validation. The three parameter sets were selected for the following two reasons. First, they exhibited firing dynamics significantly different from each other. Second, they imitate the three types of experimental conditions, i.e., administration of GABA blocker picrotoxin (PIX) and gap-junction blocker carbenoxolone (CBX) and their control (CON), in our previous study [3]. We applied the minimum-error method to estimate $g_{i}$ and $g_{c}$ from the test spike data and evaluated the estimation accuracy as the absolute error between the estimated and true parameter values.

\subsection{Feature extraction}

We investigated the spike data in terms of a feature vector (FV) composed of 67 quantities that characterize the firing dynamics. One-third of the FV components characterize temporal properties, while the rest of the components quantify spatial properties of the firing patterns.

1. The mean firing rate (FR) of spike segments was calculated as the number of spikes per $1 \mathrm{~s}$.

2. The local variation (LV) was calculated as

$$
L V=\frac{1}{R-1} \sum_{r=1}^{R-1} \frac{3\left(T_{r+1}-T_{r}\right)^{2}}{\left(T_{r+1}+T_{r}\right)^{2}}
$$

where $T_{r}(r=1,2, \ldots, R)$ is the $r$ th inter-spike interval (ISI).
3. The auto-correlogram for 20 delays (ACG 1-20) ranged between $0 \mathrm{~ms}$ and $1000 \mathrm{~ms}$ with a bin size of $50 \mathrm{~ms}$.

$$
A C G_{x, i}(\tau)=\sum_{k=1}^{K} x_{i}\left(t_{k}\right) x_{i}\left(t_{k}-\tau\right)
$$

Here, $x_{i}\left(t_{k}\right)$ represents the occurrence of spikes at the $k$ th time step in the $i$ th neuron and $\tau$ is the time delay.

4. The cross-correlogram for 20 delays (CCG 1-20) corresponding to delays of $0-50 \mathrm{~ms}, 50-100 \mathrm{~ms}, \ldots, 950-$ $1000 \mathrm{~ms}$ was computed as

$$
C C G_{x, i, j}(\tau)=\sum_{k=1}^{K} x_{i}\left(t_{k}\right) x_{j}\left(t_{k}-\tau\right)
$$

5. The minimal distance (MD1-25) was defined as a normalized distribution of

$$
s_{l}^{i, j}=1-\exp \left(\frac{-2 \min _{m}\left|t_{l}^{i}-t_{m}^{j}\right|}{\bar{d}_{j}}\right)
$$

where the distance is measured between the $l$ th spike of the $i$ th neuron and the closest spike of the $j$ th neuron. Here, $t_{l}^{i}$ is the time of the $l$ th spike of the $i$ th neuron, $\bar{d}_{j}$ is the mean ISI of the $j$ th neuron, and $m$ ranges from 1 to the total number of spikes of the $j$ th neuron. If the spike train is generated by a random process, the distribution will be uniformly distributed between 0 and 1 .

To reduce the redundancy of these features, we analyzed the FV by PCA. The top three principal components, which amount to $55 \%$ of the data variance, were selected for computing the minimum distance.

\subsection{Minimum-error method}

As a solution to the inverse problem of estimating $g_{c}$ and $g_{i}$ from test data, we searched for values of $g_{c}$ and $g_{i}$, the corresponding training data of which showed the closest match (minimum PCA distance) to the test data in the PCA space. The distance between the two data sets is defined as

$$
\operatorname{distance}(y, \hat{y})=\sqrt{\sum_{i=1}^{N}\left(y_{i}-\hat{y}_{i}\right)^{2}}
$$

where $y$ and $\hat{y}$ represent PCA scores of the training and test data, respectively, and $N=3$ is the number of selected PCA components.

\section{Results}




\subsection{Firing dynamics of simulation data}

To demonstrate typical features of the firing dynamics of the model, we show five major features of the training data. Figure 1 indicates that the features related to neuronal firing (ACG1, FR, LV) mainly conveyed information of $g_{i}$, whereas those related to synchronous firing across neurons (CCG1 and MD2) conveyed information of both $g_{i}$ and $g_{c}$. This is probably due to the fact that $g_{i}$ controls the firing in individual neurons, while $g_{c}$ controls the interaction among neurons. This result suggests that the estimation accuracy may differ between $g_{i}$ and $g_{c}$. The $g_{i}$ estimate should be more precise than that of $g_{c}$ because of the distinguishability of the $g_{i}$ variable.

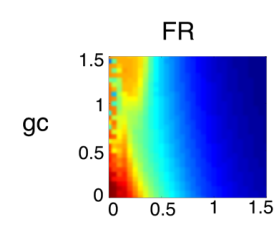

gi gi

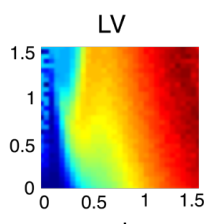

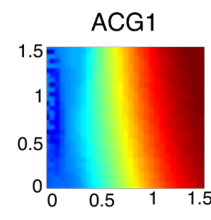

gi

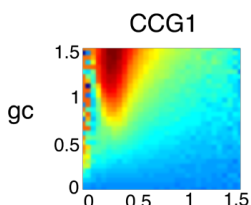

gi

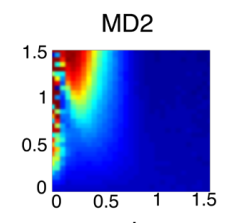

gi
Figure 1: Five major features of training data: Each mean feature value is plotted for various $g_{i}$ and $g_{c}$. The color map is normalized by the peak value

\subsection{Quantitative analysis of test data}

Raster plots are drawn in Figure 2 for spike trains in $50 \mathrm{~s}$ for three representative neurons in the test data. While neurons under condition $\sharp 1$ constantly fired at a very high frequency $(>1.5 \mathrm{~Hz}$, blue traces), the firing rates of neurons in the other two data sets were rather low $(<0.2 \mathrm{~Hz}$, orange and green traces). This should be because increasing the inhibitory synaptic strength $g_{i}$ makes the neurons fire less frequently.

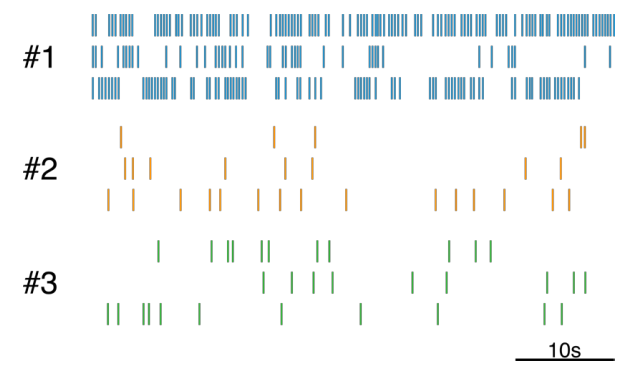

Figure 2: Spike trains of three representative neurons in three test data sets $(\sharp 1, \sharp 2, \sharp 3)$
Next, we analyzed the firing dynamics of the test data in terms of the five major features averaged over nine neurons under the three conditions $(\sharp 1, \sharp 2, \sharp 3$ ) (Figure 3 ). For three features (FR, CCG1, and MD2), condition $\sharp 1$ showed significantly higher values than for other conditions, whereas the situation was opposite for the other two features (LV and ACG1). These changes in the features indicate that the firing dynamics became more frequent and more regular in the individual neurons and more synchronous across the neurons under condition $\sharp 1$.

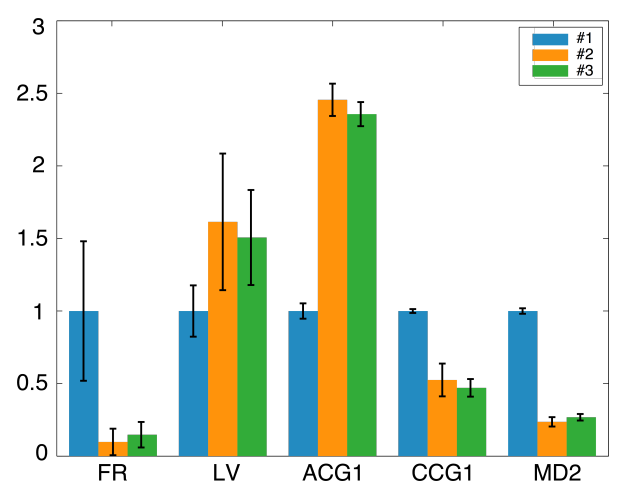

Figure 3: Five major features (FR, LV, ACG1, CCG1, and MD2) of test spike data: Each feature was normalized by the averaged feature of $\sharp 1$ data

\subsection{Parameter estimation for the test data}

We applied the minimum-error method for $50 \mathrm{~s}$ spike trains in the test data sets and separately calculated the accuracy as the absolute difference between the estimated and true parameter values of $g_{i}$ and $g_{c}$. Figure 4 shows the mean and standard deviation of the errors in $g_{i}$ and $g_{c}$ estimates for the test data. As mentioned in section 3.1, $g_{i}$ errors for all three test data sets were significantly smaller than those of $g_{c}$, being approximately $8 \%$ and $35 \%$ of the parameter range, respectively.
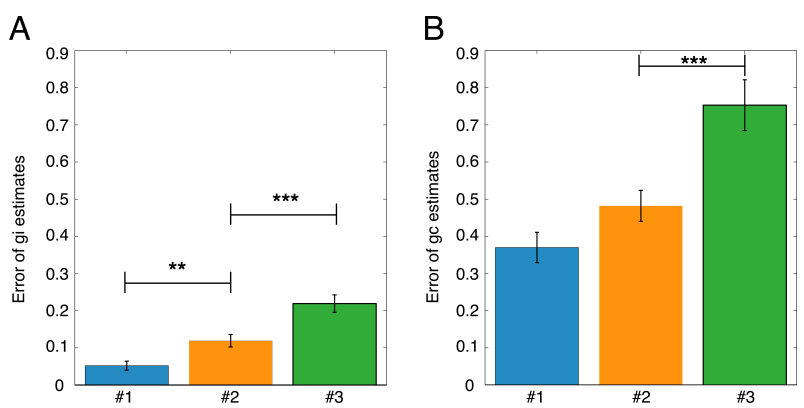

Figure 4: Errors of $g_{i}(\mathrm{~A})$ and $g_{c}(\mathrm{~B})$ estimates for nine neurons in the test data

The analysis of variance (ANOVA) indicated that there 
were significant differences of the $g_{i}$ and $g_{c}$ errors across both factors: the type of parameters $\left(g_{i}\right.$ or $\left.g_{c}\right)\left(p=1.43 \times 10^{-29}\right)$ and the parameter values among test data sets $(p=5.84 \times$ $10^{-11}$ ) (Table 1). These results indicate that the accuracy of the minimum-error method depends upon both the types and the values of the parameters to be estimated. It should be noted that the error is relatively large for the $g_{c}$ estimates. Since $g_{c}$ controls coupling among the neurons, the level of synchrony, such as CCG1, might contain useful information on the $g_{c}$ value. As indicated by CCG1 in Figure 3, the level of synchrony does not change too much among the three data sets $(\sharp 1, \sharp 2, \sharp 3)$. This might be the reason why the $g_{c}$ estimates were not particularly accurate.

Table 1: Estimation errors of $g_{i}$ and $g_{c}$ for test data

\begin{tabular}{|c|c|c|c|}
\hline & $\sharp 1$ & $\sharp 2$ & $\sharp 3$ \\
\hline$g_{i}$ & $0.05 \pm 0.01$ & $0.12 \pm 0.02$ & $0.22 \pm 0.02$ \\
\hline$g_{c}$ & $0.37 \pm 0.04$ & $0.48 \pm 0.04$ & $0.75 \pm 0.07$ \\
\hline $\mathrm{F}(\sharp 1, \sharp 2, \sharp 3)$ & \multicolumn{3}{|c|}{$25.54\left(p=5.84 \times 10^{-11}\right)$} \\
\hline $\mathrm{F}\left(g_{i}, g_{c}\right)$ & \multicolumn{3}{|c|}{$159.72\left(p=1.43 \times 10^{-29}\right)$} \\
\hline
\end{tabular}

We further investigated the effect of the spike data length on the estimation accuracy. The parameters were estimated under different settings of the data length, which was varied from $50 \mathrm{~s}$ to $500 \mathrm{~s}$. These data lengths were comparable to the recording time of the experimental data in our previous study [3]. Figure 5 indicates that increasing the spike data length enhanced the estimation accuracy for both $g_{i}$ and $g_{c}$. The amount of improvement evaluated over $500 \mathrm{~s}$ were about $50 \%$ for $g_{i}$ estimates (Figure 5A) and nearly $30 \%$ for $g_{c}$ estimates (Figure 5B) compared with those evaluated over 50 s. This result confirms that the length of $500 \mathrm{~s}$ used for the experimental data in our previous study was long enough to obtain reliable estimates.
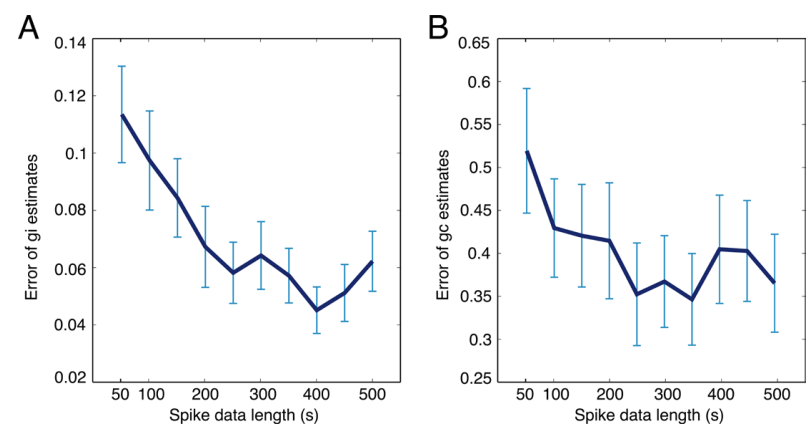

Figure 5: Dependence of the estimation error on spike data length: (A) $g_{i}$ estimates. (B) $g_{c}$ estimates

\section{Conclusions}

When there exists a huge mismatch between mathemati- cal models and the real brain, the problem of parameter estimation usually becomes severely ill-posed. The fact that the simulated neurons cannot perfectly reproduce the firing dynamics of the real neurons impairs the accuracy of parameter estimation from experimental spike data. The aim of our previous study was to overcome this challenging problem on the basis of the idea that the parameters are estimated for small segments of spike data. The distribution of the estimated parameters for small segments captured the essential features of the experimental conditions very well (PIX to reduce $g_{i}$ and CBX to reduce $g_{c}$ ) [3]. However, the estimation accuracy had not yet been properly evaluated, because the true values for $g_{i}$ and $g_{c}$ were unknown for the experiments.

In this study, we attempted to verify the minimum-error method using simulated spike data as the test data sets. Since the true values of $g_{i}$ and $g_{c}$ in the test data were known, it was straightfoward to evaluate the estimation errors. Two important results were obtained from our study. First, the accuracy of the minimum-error method was found to strongly depend on the firing pattern of the neurons. Synchronous pattern is important to detect changes in $g_{c}$, whereas irregular firing pattern is useful in detecting changes in $g_{i}$. Second, longer spike data can significantly improve the performance of the estimation. In conclusion, we argue that the minimum-error method is effective for resolving the ill-posedness of the inverse problem despite the imperfectness of the model.

\section{Acknowledgments}

The authors would like to thank Dr. Okito Yamashita, Dr. Masa-aki Sato, Dr. Mitsuo Kawato, and Dr. Keisuke Toyama from the Advanced Telecommunications Research Institute (ATR) for their support in this study. This work was supported in part by Grants-in-Aid for Scientific Research (C) (No. 23560446) from MEXT of Japan and Grants-in-Aid for JSPS Research Fellows.

\section{References}

[1] M. C. Vanier and J. M. Bower: A comparative survey of automated parameter search methods for compartmental neural models, J. Comput. Neurosci., Vol. 7, pp. 149-171, 1999.

[2] W. V. Geit, E. D. Schutter and P. Achard: Automated neuron model optimization techniques: A review, Biol. Cybern., Vol. 99, pp. 241-251, 2008.

[3] M. Onizuka, H. Hoang, M. Kawato, I. T. Tokuda, N. Schweighofer, Y. Katori, K. Aihara, E. J. Lang and K. Toyama: Solution to the inverse problem of estimating gap-junctional and inhibitory conductance in inferior olive neurons from spike trains by network model simulation, Neural Netw., Vol. 47, pp. 51-63, 2013. 